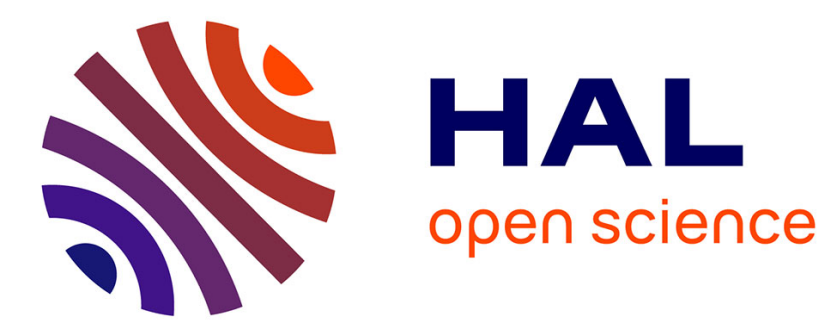

\title{
New Trends in the Design of Metal Nanoparticles and Derived Nanomaterials for Catalysis
}

Karine Philippot, Alain Roucoux

\section{To cite this version:}

Karine Philippot, Alain Roucoux. New Trends in the Design of Metal Nanoparticles and Derived Nanomaterials for Catalysis. Karine Philippot; Alain Roucoux. Nanoparticles in Catalysis: Advances in Synthesis and Applications, Wiley-VCH, pp.1-11, 2021, 9783527346073. 10.1002/9783527821761.ch1 . hal-03235252

\section{HAL Id: hal-03235252 \\ https://hal.science/hal-03235252}

Submitted on 5 Nov 2021

HAL is a multi-disciplinary open access archive for the deposit and dissemination of scientific research documents, whether they are published or not. The documents may come from teaching and research institutions in France or abroad, or from public or private research centers.
L'archive ouverte pluridisciplinaire HAL, est destinée au dépôt et à la diffusion de documents scientifiques de niveau recherche, publiés ou non, émanant des établissements d'enseignement et de recherche français ou étrangers, des laboratoires publics ou privés. 


\title{
By Alain Roucoux* and Karine Philippot*
}

\author{
Affiliation \\ Dr Karine Philippot* \\ CNRS, LCC (Laboratoire de Chimie de Coordination), UPR8241, Université de Toulouse, \\ UPS, INPT, F-31077 Toulouse cedex 4, France \\ E-mail: karine.philippot@1cc-toulouse.fr
}

ORCID : 0000-0002-8965-825X

\section{Pr Alain Roucoux*}

Univ Rennes, Ecole Nationale Supérieure de Chimie de Rennes, CNRS, ISCR - UMR6226, F-35000 Rennes, France

E-mail: Alain.Roucoux@ensc-rennes.fr

ORCID : 0000-0002-3283-8241

\section{Keywords:}

metal-based nanoparticles; transition and earth-abundant metals; water, polyols, ionic liquids; nanocellulose; magnetic silica supports; metal-organic frameworks; nanocatalysis; sustainable chemistry; biomass; water-splitting; theoretical modeling

\section{Abstract:}

The purpose of this first chapter is to introduce the content of the book "Nanoparticles \& Catalysis: Advances in Synthesis and Applications". As suggested by the title, this book is fully dedicated to the new trends in research addressing the engineering of metal nanoparticles for their application in catalysis field. After general information on the position, interests and perspectives of this over growing domain that is nanocatalysis, a short overview of the fifteen chapters gathered is provided. The so-assembled data cover different domains of nanocatalysis both in terms of nature of the nanocatalysts developed (metal nanoparticles or derived composite materials) than ofvariety catalyses and reaction media studied. The reported applications deal with either nanocatalysis in solution conditions or in supported ones. The readers from academic or industrial communities will find in this book a basic 
knowledge on the recent advances in the design of well-defined and efficient nanocatalysts by taking advantage of diverse synthesis methods and materials. This collection of chapters illustrates well the research efforts presently done in order to reach higher and original performances but also a better understanding of the key parameters for nanocatalysis.

\section{Nanocatalysis: position, interests and perspectives}

Being part of heterogeneous catalysts, metal nanoparticles have been known for a long time (see for instance the pioneering works of P. Sabatier [1] and L.D. Ramino and F.F. Nord [2]), but a renewed interest has emerged in the last three decades for the design of better defined systems [3]. Interestingly, a great part of the former heterogeneous catalysis community is now merging into the nanoparticle one [4]. Thus, as it can be observed in the literature through the over increasing number of papers and patents published by both academic and industrial institutes, huge research efforts are devoted to the synthesis of more precisely defined metal nanospecies and even more recently at an atomic precision level [5,6,7], as well as the study of their characteristics. This keen interest for nanoscale metal-based species derives from their particular matter state (finely divided metals) and their electronic parameters, thus influencing the physical and chemical properties that these entities present in comparison with bulk metals and molecular complexes. Small nanoparticles are usually called nanoclusters but there is a continuum of situations from molecules to solid state between small clusters defined by molecular orbitals and larger nanoparticles defined by energy band structures. These various types differ by the number of metal atoms, the nature of the stabilizing ligands, and the dispersity. Rigorously the term cluster or nanocluster only concerns molecularly polymetallic assemblies with ligands for which the X-ray crystal structure is known, whereas the term nanoparticles is used for mixtures of more or less polydisperse large nanoclusters defined by the histogram disclosed by transmission electron microscopy measurement [4]. Besides the fundamental aspects of the research around metal nanoparticles, the interest is also governed by the potential applications that they may find. The unique properties of nanosized metal particles make them very attractive materials for various domains of applications including optoelectronics, sensing, biomedicine, energy conversion, storage and catalysis, as non-exhaustive examples $[8,9,10,11]$. Concerning this last field, several books dedicated to nanocatalysis have been edited in the last fifteen years $[1,12]$. Indeed, metal nanoparticles are particularly interesting catalytic species due to the high surface to volume ratio they display. This ratio is promising when metal nanoparticles are at a size as close as one nanometer, or even below (subnanoparticles), since the number of surface 
atoms can be $>90 \%$ thus providing a great number of potential active sites. Remarkably, subnanometric particles of late transition metals have been reported as very active although the optimum catalytic activity is believed to be attained with particles containing between 12 to 20 atoms (i.e. sizes close to $1 \mathrm{~nm}$ or slightly below) [13]. The atomic sites exposed in metal nanoparticles may have various reactivities owing to their different coordination numbers according to their situation on the surface (corner, edge, and face atoms). By the way, the development of synthesis tools that enable to produce ultra-small nanoparticles remains of prime importance in order to promote high surface area combined with an efficient distribution of surface atoms. Indeed, the proportion of edge and corner surface atoms possessing lower coordination numbers increases with the decrease in the particle size. Besides the size, other key morphological parameters need to be controlled. Thus, the crystalline structure is also of great importance since according to the types of crystalline plans that can be exposed at the surface, different catalytic properties could be achieved. Controlling the particles shape also constitutes another route to orientate the crystalline plans exposed $[14,15,16]$. The last but not the least key-parameter is the composition of the metal nanoparticles that has to be adjusted depending on the targeted catalytic reaction. Apart from the nature of the metallic core that may govern the reactivity (some metals are well-known for certain catalytic applications but not for others), the surrounding stabilizer for colloidal catalysis (ionic liquids (ILs), polymers, dendrimers, surfactants, polyols, ligands, etc.) or the support for supported catalysis (metal oxides such as silica, metal organic frameworks (MOFs), carbon derivatives, nanocelluloses, etc.) may also influence or even orientate the catalytic performances. While calcination is typically applied in traditional heterogeneous catalysis in order to suppress any organics and liberate the active sites, such treatment on small nanoparticles can be critical, thus potentially leading to their sintering. Moreover, naked nanospecies are not always optimal catalysts. In modern nanocatalysis, the presence of organic ligands onto the particles surface is not considered as detrimental for catalytic applications but could be a way to improve or even modify the chemoselectivity [17]. Aiming at catalysis by appropriate design, current developments in nanochemistry dedicated to catalytic applications often rely with multifunctionality [18]. This can be achieved by the proper design of nanohybrids, the term hybrid referring to the appropriate association between a metal core and a stabilizing shell or a support. When using typical ligands from coordination chemistry as capping agents, a parallel can be performed with molecular catalysis. Indeed, the interaction of the ligands with the metal atoms on the particle surface can be compared to ligand interactions with the metal centers in homogeneous complexes, and is a parameter of 
paramount importance for stability and catalytic performances (activity and selectivity properties). Thus, ligands can be chosen in order to tune the surface properties of metal nanoparticles through steric and/or electronic effects $[19,20]$. The challenge remains to find protective agents able to stabilize well-defined nanoparticles while controlling accessibility at the metal surface and reactivity [17,21]. Strongly bound capping ligands (like thiols or phosphines) can result in the poisoning of a nanocatalyst at high surface coverage. But the presence of limited amount of ligand can be beneficial in terms of catalytic performances (suitable and replicable activities and/or selectivities). The strong coordination of a ligand at a metal surface can also be a way to block selectively some active sites in order to orientate the catalysis evolution. In comparison with the investigation of facet dependency $[16,22]$, the ligand influence on the catalytic activity has been less intensively studied but recent results illustrate well the interest to do so [23, 24,25, 26,27]. Capping agent-stabilized metal nanoparticles can be applied to catalysis as stable colloidal suspensions in various media (water, polyols, organic solvents) but also in heterogeneous conditions after their deposition on the surface or confinement in the pores of solid supports [28]. Ionic liquids [29] are also very efficient to stabilize metal nanoparticles and their colloidal suspensions can even be deposited onto inorganic materials [30].When employing a support, not only it prevents nanoparticle aggregation but also it may act in synergy with nanoparticle surface and favor the activation of substrates in a manner comparable to the positive synergy observed between two transition metal atoms in alloys or between a transition metal and a main group element like nitrogen. So, it was found that $\mathrm{N}$-doped carbon supports were superior to undoped analogues due to such synergy effects [4].

Thus, having in hand synthesis strategies that allow access, in a reproducible manner, to welldefined metal nanoparticles in terms of size, crystalline structure, composition (metal cores and stabilizing agents), chemical order (bimetallic or ternary systems), shape and dispersion constitutes a beneficial condition to finely investigate the catalytic properties of metal nanoparticles and define structure/properties relationships. Taking advantage of recent developments in nanochemistry that offer efficient tools to reach these objectives, nanocatalysis is now well-established as a borderline domain between homogeneous and heterogeneous catalysis. With a molecular approach, nanocatalysts can be considered as assemblies of individual active sites where metal-metal bonds will have also influence [31]. Precisely designed nanohybrids (including the choice of an adequate and non-innocent stabilizer or support) are expected to present benefits from both homogeneous and heterogeneous catalysts, namely high reactivity and better selectivity [32]. One aim lies in the 
design of more performant nanocatalysts in order to develop more efficient and ecocompatible chemical production [33]. If huge progress has been performed in the last decade, this topic still remains challenging, in particular with the crucial need to develop multigramscalable catalytic routes presenting a reduced environmental footprint and economically viable cost for industrial applications. Furthermore, model catalysts are also needed in order to better understand the relationship between the characteristics of metal nanoparticles and their catalytic performances and thus bridge the gap between model surfaces and real catalysts. Each progress that contributes to reduce the gap of knowledge between nanocatalysts and homogeneous catalysts constitutes a step forward the development of more efficient catalytic systems. Efforts have thus to be pursued in order to one day be able to anticipate the design of suitable catalysts for a given reaction.

Various metals are investigated in nanocatalysis towards these principles, with a huge number of studies dedicated to gold which is highly reputed for $\mathrm{CO}$ oxidation and emerges now in hydrogenation catalysis [34,35], or palladium as relevant metal for various C-C coupling reactions and also catalytic hydrogenation [36,37]. Other metals like rhodium, ruthenium, platinum, iridium, nickel, cobalt, and iron among others, are also the subject of numerous studies. Some of these metals, generally called earth-abundant such as iron and cobalt or more recently manganese or copper have attracted increasing attention in the field of nanocatalysis due to their low cost, ready availability, low toxicity, greater sustainability [38,39]. Indeed, for many traditional catalytic transformations and challenging reactions, earth-abundant transition metal nanoparticle catalysts have already appeared as attractive alternatives to noble ones.

The purpose of this book is to provide the readers a synthetic overview of the recent advances in research that address the investigation of well-controlled metal nanoparticles in the domain of catalysis in suspension conditions (colloidal catalysis) and supported conditions.

\section{Metal nanoparticles: what is new?}

As mentioned above, nanocatalysis, being an exciting research area at the frontier between homogeneous and heterogeneous fields, has known a huge evolution during the past 25 years. This great interest mainly derives from the original catalytic properties that nanoparticles offer but also to the great advances in their synthesis in solution (aqueous, organic, glycerol, polyols, or ionic liquids, as main examples) or onto supports. Also the combination of complementary analytical techniques from both molecular chemistry and solid chemistry together with development of computational chemistry tools for the study of their surface 
state and/or modeling account for this still growing interest. Worlwide, numerous scientists are involved in both "nanochemistry" and "nanocatalysis" with a main and common objective, the design of well-controlled nanoparticles in terms of size, dispersion and surrounding environment to afford high and/or original catalytic performances.

Compared to previous methodologies in which metal nanoparticles were produced almost exclusively via heterogeneous approaches (top-down approach), current strategies offer a better control of the nanoparticle characteristics (bottom-up approach). Nanometer-sized transition metal particles for catalysis, usually called "nanocatalysts", are structure-sensitive; their properties in terms of activities and selectivities depend on their core-size, shape, morphologies as well as nature, composition and their support materials. Nanocatalysis includes the development of metallic and metal oxide nanoscale materials with defined structure and morphology and their use as active species or as supports of the active phase.

This book will provide to the readers a basic knowledge on the current trends in the domain of metal nanoparticle engineering and of derived composite materials, all the research efforts done aiming to reach better catalytic performances but also a better understanding of the keyparameters in nanocatalysis. A large range of data from the synthesis of metal nanoparticles, either in solution or deposited onto various supports, to their application as nanocatalysts in diverse catalytic reactions have been collected. Actually, each chapter of this book proposes a critical overview on the concerned subject through the discussion of relevant examples from the recent literature.

The 15 fifteen chapters of this book cover a wide range of subfields by exploring in detail a great variety of nanocatalysts, catalytic media, catalytic reactions and potentially their external activation. Following two introductive chapters dedicated to general interests of metal nanoparticles and their position between molecular and heterogeneous catalysts, four thematic parts structure this book (Figure 1). The first two parts concern nanoparticles as active species in solution (Part I) or deposited onto supports (Part II). Part III is devoted to sustainable applications for energy production or upgrading of renewables. In Part IV, recent advances in physical activation of nanocatalysts for avoiding classical heating are first illustrated. Then, examples on the contribution of computational chemistry dedicated to metal nanostructures are discussed. This subdomain provides theoretical insights and modelings of nanocatalysts for a better understanding of the metal surface environment and of catalytic reactions that occur there. 


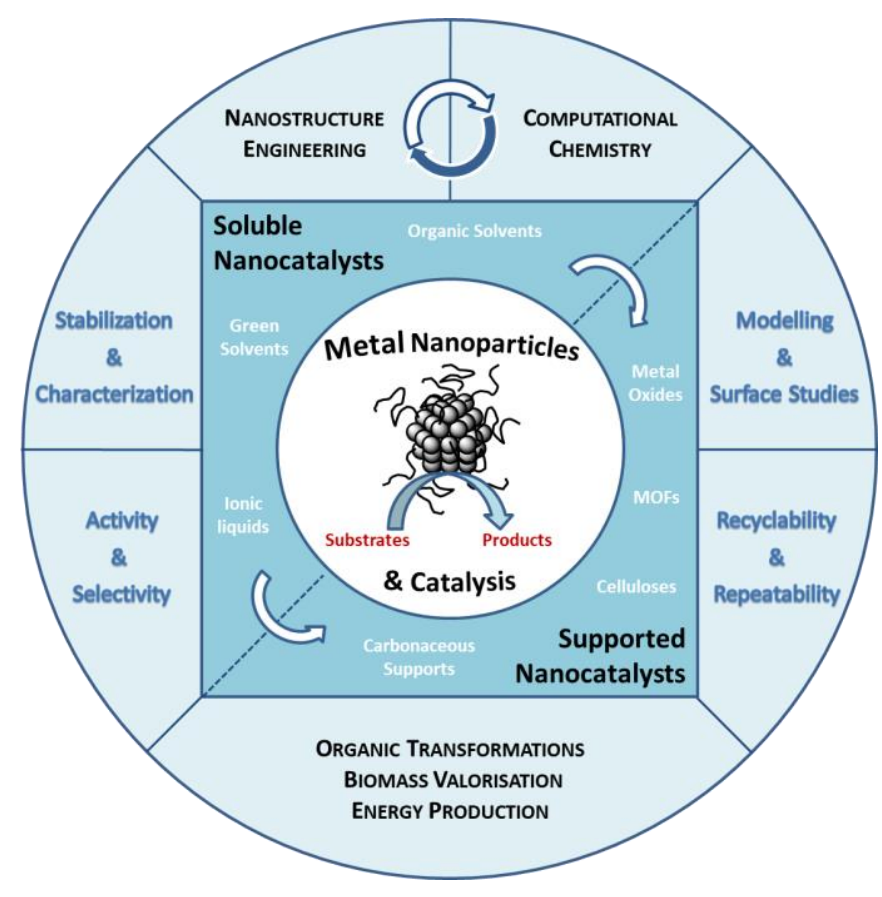

Figure 1. Schematic overview of the main but not exhaustive trends currently developed today in the area of nanocatalysis and illustrated through the chapters' contents of this book

The Chapter 2 constitutes a strategic introduction on the nature of nanoparticle-based catalysts at the interface between molecular complexes and heterogeneous systems. Based on experimental results issued from several analytical techniques such as electron microscopy, mass spectrometry, mercury tests, etc., this chapter reports on the difficulties to reveal the true nature of the active species in dynamic catalysis. According to the catalytic applications, nanoparticles, nanoclusters, mononuclear complexes, and multicenter metal complexes can be simultaneously present providing cocktail-type systems detailed in a cocktail of metal species and cocktail of catalysts.

The following four chapters are devoted to nanoparticles in solution. In Chapters 3 and 5, the synthesis of nanoparticles in polar and green solvents (water and polyols) is described. In pure water, several applications in biphasic conditions are reviewed including hydrogenation, oxidation and C-C coupling reactions based on the stabilization of nanoparticles with watersoluble ligands, surfactants and polymers (Chapter 3). All approaches in the reported chemical transformations are described under the angle of environmentally-friendly and economically viable processes. Polyol media such as ethylene glycol or glycerol, can act as multi-task components (solvents, reductants, stabilizers) allowing bottom-up strategies called "polyol synthesis" for the synthesis of stable and well-defined nanomaterials. Chapter 5 is mainly centered on metal-based nanoparticles in polyol media synthesized by chemical 
approaches but the synthesis of metal nanoparticles by sputtering techniques (Top-down methodologies) is also introduced. Chapter 4 reports the synthesis of transition metal nanoparticles in organic phase based on the decomposition of metalloorganic complexes through reduction or ligand displacement from the metal coordination sphere under mild conditions. This synthesis approach allows reaching very well-defined metal nanoparticles that can be used as model systems for a better understanding of the structure/properties relationships. Thus, the influence of the capping-ligands onto the surface properties of metal nanoparticles and consequently on their catalytic performance is discussed through a variety of examples related to hydrogenation catalysis. The formation and stabilization of various transition metal nanoparticles in ionic liquids (ILs) and their applications in catalysis under homogeneous and multiphase conditions are described in Chapter 6. Recent investigations of these soluble nanoparticles in hydrogenation of carbon dioxide as well as in the $\mathrm{C}-\mathrm{C}$ coupling reaction and the hydroformylation of alkenes are highlighted.

The subsequent four chapters are dedicated to metal-based nanoparticles deposited onto a broad variety of supports. In Chapter 7, the interest of cellulose nanocrystals (CNCs) and cellulose nanofibers (CNFs) to support metal NPs is demonstrated, evidencing that this new trend is an active and promising field of research in catalysis. In a sustainable context, nanocellulose derivatives available from biomass prove to be pertinent as renewable supports for the direct deposition and anchoring of metal nanoparticles. The use of unmodified as well as functionalized CNCs is highlighted in catalytic C-C coupling and reduction reactions. Chapter 8 describes the preparation of magnetically recoverable supported metal nanocatalysts. The deposit of various metal nanoparticles is detailed according to the nature of precursors (salts, organometallic complexes or pre-formed nanoparticles). The so-produced active species either supported onto simple iron oxide or coated with a protective layer (carbon, silica or other oxides) exhibit excellent magnetic properties and exciting catalytic activities. Factors influencing the synthesis of metal nanoparticles supported by TEM images as well as their catalytic performances due to the nanoparticle environment (impact of ligands) are discussed into detail. In chapter 9, recent studies on the synthesis of metalorganic frameworks (MOF)-supported nanoparticles are reviewed. First, it focuses on the most common methods based on a "bottom-up" approach to generate different types of colloidal metal nanoparticles. Second, the synthetic methods of MOFs are summarized followed by the integration methods developed to integrate metal nanoparticles into MOFs. This chapter not only provides a good understanding of the concerned topic but also the advantages versus drawbacks of respectives methods are highligted. Catalytic applications of 
several typical metal nanoparticles/MOF composites based on their dimensional properties and structure-property relationships are fully discussed. Focused on $\mathrm{Pt}$ nanoparticles supported onto SBA-15, Chapter 10 presents synthetic methodologies to deposit metal nanoparticles over silica. Interestingly, how this support can lead to superior activity as the result of tunning of the pore sizes, their inter-connection or surface area for a selection of reactions is understandingly discussed. These heterogeneous nanocatalysts provide high catalytic activity in many reactions. Such as oxidation, hydrogenation and carbon-carbon coupling reactions as reported in detail.

After the review of typical synthesis of nanoparticle suspensions in various solvents in chapters 3-6 and their deposit or inclusion in different supports (chapters 7-10), the next three chapters are devoted to nanocatalysis. They focus on recent applications and key-reactions for chemical and energy transformations including the production of hydrogen by dehydrogenation of formic acid or water-splitting as well as eco-respectful aspects with the transformation of biomass and $\mathrm{CO}_{2}$ into fuels or added-value chemicals. Thus, Chapter 11, concerns supported nanocatalysts for the hydrogenation of $\mathrm{CO}_{2}$ into fine oxygenated chemicals such as methanol, dimethyl ether or formic acid. The mechanisms as well as new opportunities and challenges for further advancing in this research field are outlined. Relying with sustainable energy sources challenge Chapter 12 reports on the use of ruthenium-based nanomaterials as catalysts for hydrogen production from water. In comparison with platinum catalysts, ruthenium metal or metal oxide nanoparticles have recently become widespread for the hydrogen evolution reaction (HER). This chapter highlights the key chemical factors that govern the HER performances and the most promising electrochemical and photochemical systems are critically discussed in order to point to possible future research directions. In the strategic context of the chemical hydrogen storage, the Chapter 13 reviews recent advances for hydrogen generation through the catalytic liquid-phase dehydrogenation of formic acid in the presence of nanocatalysts including monometallic or polymetallic nanoparticles. Their advantages versus disadvantages are discussed according to factors impacting their catalytic performances in terms of activity, selectivity and durability.

The last two chapters are devoted to recent advances in the light of the new activation methodologies and theoretical modeling developments. The Chapter 14 presents the magnetic induction and heating performances of adapted nano-scaled iron alloys to perform catalysis. This recent advance is based on the use of nanoparticles playing simultaneously the role of heating agent through magnetic hyperthermia and the role of the catalyst for a large scope of 
reactions based on activation of $\mathrm{CO}, \mathrm{CO}_{2}$ and light alkanes. Thanks to a fine control of the magnetic properties of nanomaterials, the perspectives of improvement of these systems are promising and constitute a future active research field. The last chapter $\left(\mathrm{N}^{\circ} 15\right)$ provides theoretical insights in metal nanocatalyst field as the result of recent progresses in computational catalysis and nanochemistry. Based on the isotropic H/D exchange and the hydrogen evolution reaction (HER) activated by ruthenium nanoparticles, this contribution is a concrete case of the positive impact of computational calculations for a better understanding of the effect of the direct environment of metal nanoparticles and the reactivity changes induced by ligands in nanocatalysis.

\section{Conclusions and Perspectives}

Being dedicated to students, young researchers and confirmed ones, this book gives a comprehensive overview on the recent advances and current trends in the engineering of metal nanoparticles - including their design, synthesis and characterisation by state-of-the-art techniques - together with their various applications in catalysis. A collection of short chapters is proposed to make the reading of the whole book easier and more dynamic, while providing the main key-aspects of the use of metal nanoparticles in catalysis today. Coming from a large range of institutions in different countries, the authors have been chosen for the originality of the synthesis method or of understanding approach or/and the interest toward a target catalytic application that they develop.

This book brings together the most recent achievements in the development of metal nanoparticles as catalysts by covering a selection of key-aspects in this challenging domain and underlying new trends. Thus, each chapter illustrates one emerging domain of nanocatalysis with concrete applications and understanding of metal nanoparticles behavior through the presentation of the most relevant examples from the recent literature including those of the invited groups.

As it can be seen in most of the chapters of this book, a cleaner chemistry and sustainable developments are at the center of current research efforts. The use of green solvents (for instance water, polyols, glycerol and in a certain extend ionic liquids) as synthesis or/and reaction media has already shown to be efficient to provide highly performant nanocatalysts for diverse organic transformations. Also, the study of catalyst lifetimes (in terms of recovering, recycling, durability and repeatability) is among the objectives of many present works. Concerning the catalytic performances, if highest possible activities are always targeted, high selectivities (both chemo- and stereo-selectivity) have become a key-point in 
order to limit as possible product separation steps which are often costly regarding solvent and energy consumption. Also, the development of nanocatalysts based on earth abundant and cheap metals is more pertinent nowadays. This allows to face the decrease of noble metal reserves on the Earth and to reduce the catalyst costs. This is particularly relevant with regards to the scale-up of catalysts for industrial processes. Beside the objective of catalyst cost reduction, a synergy effect can be expected by associating earth-abundant metals to noble ones. The utilization of biosourced materials (like celluloses or biochars) to build catalysts, of biosourced chemicals as new reaction media (such as lactate esters e.g. ethyl lactate, MeTHF) or of abundant and cheap biorenewables substrates (like terpenes, carbohydates or agricultural residues, vegetable oils among others) as platforms of new synthons by organic transformations has known a hudge development in nanocatalysis these last years with promising achievements. This allows not only to take advantage of natural chemicals but also to valorize certain wastes issued from biomass (for instance woody cellulose, chitin from shellfish shells, etc.). Another important development is the use of raw chemicals issued from chemical industry (like $\mathrm{CO}_{2}$ ) or resulting from the increasing human activities. Concerning the improvement of catalytic transformations which are at the basis of the production of energy vectors like hydrogen production (either directly from water through the watersplitting process or from dehydrogenation of formic acid that can derive from $\mathrm{CO}_{2}$ ), among other present challenges in this domain, nanochemistry already proved to bring powerful solutions. The transformation of $\mathrm{CO}_{2}$ into added-value chemicals and fuels is a particularly difficult task where metal nanocatalysts did not really emerge so far but recent results are very encouraging and novel nanoscale catalytic systems may meet this challenge in the near future. The application of original heating systems (hot-spots obtained by magnetic induction or plasmonic excitation via the use of adequate nanocatalysts) is another strategy to limit energy consumption in catalytic organic transformations. Recent progresses in the domain of computational chemistry methods applied to metal nanoparticles proved these approaches to be very powerful and to provide also a high level of accurency in nanocatalysis.

All these strategies provide promising perspectives and thus merit to be largely explored in order to find solutions to present challenges that chemistry world is facing or/and to open new research avenues for anticipating futur ones..

\section{References}

[1] Sabatier P., in La Catalyse en Chimie Organique, C. Béranger (Ed.), 1913. 
[2] Rampino, L. D.; Nord, F. F. J. Am. Chem. Soc. 1941, 63, 2745-2749.

[3] Serp, P. and Philippot, K. (Eds), Nanomaterials in Catalysis. Wiley-VCH, Weinheim, Germany, 2013; p 494.

[4] Astruc D., Chem. Rev. 2020, 120, 461-463.

[5] Jin, R.; Zeng, C.; Zhou, M.; Chen, Y., Chem. Rev. 2016, 116, 10346-10413.

[6] Jin, R.; Pei, Y.; Tsukuda, T., Acc. Chem. Res. 2019, 52, 1-1.

[7] Kang X, Zhu, M. Chem. Mater. 2019, 31, 9939-9969.

[8] Schmid, G.(Ed.), Nanoparticles: From Theory to Application, Second Edition. WileyBlackwell: 2010; p 522.

[9] Talapin, D. V.; Shevchenko, E. V., Chem. Rev. 2016, 116, 10343-10345.

[10] Wu, L.; Mendoza-Garcia, A.; Li, Q.; Sun, S., Chem. Rev. 2016, 116, 10473-10512.

[11] Gilroy, K. D.; Ruditskiy, A.; Peng, H.-C.; Qin, D.; Xia, Y., Chem. Rev. 2016, 116, 10414-10472.

[12] a) Zhou, B.; Han, S.; Raja, R.; Somorjai, G. A. (Eds.), Nanotechnology in Catalysis, Springer, New York, 2003; b) Heiz, U.; Landman, U.; Eds., Nanocatalysis. Springer, Berlin, Heidelberg: 2007; c) Roucoux, A.; Philippot, K., Homogeneous Hydrogenation: Colloids Hydrogenation with Noble Metal Nanoparticles. In The Handbook of Homogeneous Hydrogenation, Vries, J. G. d.; Elsevier, C. J. (Eds.), Wiley-VCH, Weinheim, Germany 2007; c) Astruc, D.(Ed.), Nanoparticles and Catalysis. Wiley Interscience New York, 2008; Corain, B.; Schmid, G.; Toshima, N.(Eds.), Metal Nanoclusters in Catalysis and Materials Science: The Issue of Size Control. Elsevier Science: Amsterdam, The Netherlands, 2008; d) R. Richards, R. K., K. Klabunde, L. Erickson ; Eds., Nanoscale Materials in Chemistry: Environmental Applications, . ACS Publications: Washington D.C., 2011; e) Polshettiwar, V.; Asefa, T.(Eds.), Nanocatalysis: Synthesis and Applications, Wiley-VCH, Weinheim, Germany 2013; f) Tao, T. (Eds.), Metal Nanoparticles for Catalysis: Advances and Applications RSC,2014; g) Prechtl, M. H. G.(Eds.), Nanocatalysis in Ionic Liquids. WileyVCH, Weinheim, Germany 2016; h) Voorde, M. V. d.; Sels, B. (Eds.), Nanotechnology in Catalysis: Applications in the Chemical Industry, Energy Development, and Environment Protection. Wiley-VCH, Weinheim, Germany 2017.

[13] Imaoka T., Kitazawa, H.; Chun W.-J., Yamamoto K., Angew. Chem., Int. Ed. 2015, 54, 9810-9815.

[14] Tao, A. R.; Habas, S.; Yang, P., Small 2008, 4, 310-325.

[15] Xia, Y.; Xia, X.; Peng, H.-C., J. Am. Chem. Soc. 2015, 137, 7947-7966. 
[16] Chen, G.; Zhang, J.; Gupta, A.; Rosei, F.; Ma, D., New J. Chem. 2014, 38, 1827-1833.

[17] Chen, T.; Rodionov, V. O., ACS Catal. 2016, 6, 4025-4033.

[18] Boles, M. A.; Ling, D.; Hyeon, T.; Talapin, D. V., Nat. Mater. 2016, 15, 141-153.

[19]Zhukhovitskiy, A. V.; MacLeod, M. J.; Johnson, J. A., Chem. Rev. 2015, 115, 11503 11532.

[20] Heuer-Jungemann, A.; Feliu, N.; Bakaimi, I.; Hamaly, M.; Alkilany, A.; Chakraborty, I.; Masood, A.; Casula, M. F.; Kostopoulou, A.; Oh, E.; Susumu, K.; Stewart, M. H.; Medintz, I.

L.; Stratakis, E.; Parak, W. J.; Kanaras, A. G., Chem. Rev. 2019, 119, 4819-4880.

[21] Yuan, Y.; Yan, N.; Dyson, P. J., ACS Catal. 2012, 2, 1057-1069.

[22] Zhou, K.; Li, Y., Angew. Chem., Int. Ed. 2012, 51, 602-613.

[23] Dykeman, R. R.; Yan, N.; Scopelliti, R.; Dyson, P. J., Inorg. Chem. 2011, 50, 717-719

[24] Gonzalez-Galvez, D.; Nolis, P.; Philippot, K.; Chaudret, B.; van Leeuwen, P. W. N. M., ACS Catal. 2012, 2, 317-321.

[25] Stratton, S. A.; Luska, K. L.; Moores, A., Catal. Today 2012, 183, 96-100.

[26] Baker, L. R.; Kennedy, G.; Krier, J. M.; Van Spronsen, M.; Onorato, R. M.; Somorjai, G. A., Catal. Lett. 2012, 142, 1286-1294.

[27] Axet, M. R.; Conejero, S.; Gerber, I. C., ACS Appl. Nano Mater. 2018, 1, 5885-5894.

[28] Costa, N. J. S.; Rossi, L. M., Nanoscale 2012, 4, 5826-5834.

[29] Janiak, C., Chapter 11 in Catalysis in Ionic Liquids: From Catalyst Synthesis to Application, Hardacre, C.; Parvulescu, V.,) (Eds.) The Royal Society of Chemistry, 2014; pp 537-577.

[30] Claus, P.; Schwab, F., Chapter 6 in Catalysis in Ionic Liquids: From Catalyst Synthesis to Application, Hardacre, C.; Parvulescu, V. ( Eds.) The Royal Society of Chemistry, 2014; pp 391-409.

[31] An, K.; Alayoglu, S.; Ewers, T.; Somorjai, G. A., J. Colloid Interface Sci. 2012, 373, 113.

[32] Somorjai, G. A.; Li, Y.-M., Top. Catal. 2010, 53, 311-325.

[33] Freund, H.-J.; Somorjai, G. A., Catal. Lett. 2015, 145, 1-2.

[34] Bond, G. C.; Louis, C.; Thompson, D. T., Catalysis by Gold. World Scientific: 2006.

[35] Louis, C.; Pluchery, O., Gold Nanoparticles for Physics, Chemistry and Biology. World Scientific: 2012.

[36] Saldan, I.; Semenyuk, Y.; Marchuk, I.; Reshetnyak, O., J. Mater. Sci. 2015, 50, $2337-$ 2354. 
[37] Chen, A.; Ostrom, C., Chem. Rev. 2015, 115, 11999-12044.

[38] Kaushik, M., Moores, A. Curr. Op; Green. Sustainable Chem. 2017, 7, 39-45.

[39] Wang, D., Astruc, D. Chem. Soc. Rev. 2017, 46, 816-854. 